\title{
Ein Fall von Erythema scarlatiniforme desquamativum mit protrahirtem Verlaufe.
}

\author{
Von \\ Dr. Ludwig Török (Budapest).
}

In Folgendem theile ich die Krankengeschichte eines Falles von Erythema scarlatiniforme desquamativum (Dermatitis exfoliativa acuta benigna) mit. Es veranlassen mich hiezu verschiedene Gründe. Der erste ist, dass die Wiener Schule lange Zeit hindurch das Studium der mit Hautröthe verbundenen, desquamativen Dermatitiden vernachlässigte, dass daher in den Ländern, in welchen diese Schule bis in die letzte Zeit mit fast uneingeschränkter Gewalt herrschte, die bessere Erkenntniss dieser Dermatosen erst in den letzten Jahren aus Frankreich vordringt. Unter solchen Verbältnissen kann es nur von Vortbeil sein, wenn die Fälle von desquamativen Dermatitiden, welche von dem bisher in Deutschland und Oesterreich-Ungarn allein anerkannten Typus der Hebra'schen Pityriasis rubra abweichen, publicirt werden. Ein zweiter Grund für die Publication des Falles ist der Umstand, dass in dem Verlaufe desselben sich an den Nägeln und Haaren Veränderungen einstellten, wie sie zu den weniger häufigen Erscheinungen des Erythema scarlatiniforme desquamativum gehören und dass der Fall sich auch durch seine längere Dauer von den gewöhnlich in 4-6 Wochen, Maximum in zwei Monaten (Brocq) ablaufenden Anfällen dieser Archiv f. Dermatol. u. Syphil. 1893. 
Dermatose unterschied. Endlich bestimmt mich zur Publication die Thatsache, dass in diesem Falle die Hautkrankheit aufgetreten war, ohne dass der Patient irgendwelche Arzneimittel zu sich genommen oder auf seine Haut applicirt hatte, während doch von verschiedener Seite betont wurde, dass das Erythema scarlatiniforme immer durch die Ingestion oder Application von Droguen hervorgerufen werde.

S. J., 50 Jahre alt, Taglöbner, stellte sich am 21. April 1892 in der Poliklinik der allg. Arbeiter-Kranken- und Invalidencasse vor. Derselbe war bisher immer vollkommen gesund gewesen. Weder bei ihm, noch bei seiner Familie waren je Hautkrankheiten vorgekommen. Auch unmittelbar vor dem Ausbruch des gegenwärtigen Leidens befand er sich vollkommen wohl und er bestreitet lebhaft, Medicamente zu sich genommen zu haben. Die Hautkrankheit, mit welcher er behaftet ist, war vor etwa 14 Tagen aufgetreten, nachdem er sich zwei bis drei Tage vorher beim Rasiren das Kinn geritzt hatte. Die geritzte Stelle hat er bloss gewaschen und kein Medicament daranf applicirt. Ein bis zwei Tage später machte sich das Gefühl von Spannung im Gesichte bemerkbar und nach weiteren 1-2 Tagen war sein Gesicht lebhaft roth („roth wie eine Mobublume" nach Aussage des Patienten). Einige Tage später trat an die Stelle der rothen eine weisse Farbe und die Haut fing an zu schuppen. Mittlerweile hatte sich die Krankheit vom Gesichte auch auf den übrigen Körper verbreitet und war im Verlaufe einer Woche vom Kopfe bis zu den Füssen und Händen angelangt. Dabei war das Allgemeinbefinden fortwährend ein gutes; dem Ausschlage waren keine prodromalen Erscheinungen, kein Frostschaver, keine Abgeschlagenheit, keine gastrischen Symptome u. s. w. vorangegangen; er hatte keine Schlingbeschwerden, keinen Schnupfen gehabt. Jucken und Brennen war keines vorhanden, bloss nach dem Waschen machte sich immer ein Gefühl von Spannung bemerkbar.

Status praesens. Die Hautkrankheit besteht in Röthe und Schuppung. Auf der behaarten Kopfhaut ist eine überaus reichliche, sehr feine, mehlartige Abschuppung vorhanden, welche sich auf Kratzen steigert. Hinter den Ohren, über dem Processus mastoideus, an der Grenze des behaarten Kopfes befindet sich eine ans weissen, feinen, kleienartigen Schuppen zusammengesetzte dicke Lage, welche sich, etwas an Dicke abnehmend, nach vorne bis zu der Stirnhaargrenze fortsetzt. Eine ähnliche Lage befindet sich vor den Ohren, über dem oberen Theile des aufsteigenden Unterkieferastes, in den Augenbrauen, sowie im Schnurrbart und in den spärlichen Barthaaren am Kinne. Das Gesicht, der Hals und die Ohren sind leicht gedunsen, diffus und lebhaft roth und zeigen eine feine Abschilferung, welche besonders in den Furchen ausgesprochen ist und dieselben als silberweise Streifen erscheinen lässt. $\mathrm{Zu}$ beiden Seiten der vorderen Halsregion gibt es je eine kindeshand- 
Ein Fall v. Eryth. scarlatinif. desquamat. mit protrahirt. Verlaufe. 581

grosse Stelle von normaler Hautfarbe; aber auch an diesen macht sich eine feine Schuppung bemerkbar; dieselbe ist schwächer ausgeprägt als an den gerötheten Hautpartien. Dabei erscheint die Haut hier leicht gerunzelt.

Die Haut auf der Brust und auf dem Rücken ist in der ganzen Ausdehnung dieser Regionen diffus und intensiv rotb. Auf dem lebhaft rothen Grunde zerstreut sind einige dunklere, leicht bläulich-rothe Flecke bemerkbar. Ueberall ist hier eine feine Absebilferung vorhanden. Die Streckfläche beider Oberextremitäten ist diffus roth, die Abschuppung geschieht hier in etwas grösseren, lamellösen Schüppchen. Diese haben in der Quer- und Längsrichtung ie 1-2 $\mathrm{Cm}$. im Durchmesser und haften entweder mit einem Rande oder mit ihrer Mitte an der darunter liegenden Epithelschichte. Nach ihrer Desquamation bildet die benachbarte Hornschichte einen weisslichen Rand um die Stelle, wo die Schüppchen früher sassen. Die Haut ist unter den Schuppen glatt roth; durch Kratzen lassen sich aber wieder weisse Hornlamellehen loslösen. Am Vorderarme nimmt die Grösse der Schuppen noch um ein Geringes zu. Etwa drei Querfinger breit aberhalb des Handgelenkes hört die diffuse Röthe der Streckfäche der Oberextremität auf. Auf der Beugeseite der Oberextremität finden sich pfennig- bis thalergrosse, unregelmässig geformte, lebhaft rothe Flecke regellos zerstreut. Diese schilfern in ähnlicher Weise, wie die Streckseite der Arme, doch ist die Schuppung hier weniger intensiv. Auf den Händen ist die Hornschichte hauptsächlich über dem Thenar und unterhalb des Handgelenkes weisslich, etwas verdickt und löst sich in grösseren Lamellen ab. Die Haut unter ihnen ist roth. Aehnliche Veränderungen zeigen sich an mehreren pfennig- bis thalergrossen Flecken beider Handrücken. In den Interphalangealräumen, an den einander zugekehrten Flächen der Finger und in den Beugen der Interphalangealgelenke ist die Haut roth und glatt; bloss an dem Rande dieser rothen Stellen sieht man einige $\mathrm{Cm}$. breite, mit der benachbarten Hornschichte zusammenhängende Hornläppchen überhängen.

Es ist zu bemerken, dass an einzelnen Stellen der oberen Extremität, in ausgebreiteter Weise jedoch auf dem Rücken die Schuppung an den Follikeln auftritt, indem an der Austrittsstelle der Lanugohärchen - längere Haare besitzt der Patient auf dem Stamme und den Extremitäten nicht - je ein kleines Schüppchen erscheint. .

Auf der Haut des Bauches und der Sacralgegend ist keine diffuse Röthe vorhanden, bloss unregelmässig geformte pfeuniggrosse, rothe Flecke mit feiner Schilferung. Ueber beiden Glutaeen je ein flachhandgrosser, unregelmässig geformter, rother Fleck mit intensiver, feiner weisser $\mathrm{Ab}$ schuppung.

Die Haut des Penis ist von normaler Farbe, gegen den Hodensack zu leicht schilfernd. Das Scrotum ist blassroth und mit feinen Schuppen bedeckt. 
Die unteren Extremitäten sind von der Krankheit weniger intensiv befallen. Die Streckfäche des Oberschenkels und die Knien sind von blassrother Farbe, der erstere in seiner unteren Partie mit feinen, weissen pulverartigen Schuppen besäet, die Gegend der Patella, in der Ausbreitung eines Silberguldens, von dicker, gräulicher fest haftender Hornschichte bedeckt. Auf dem Unterschenkel einige wenige blassrothe, schilfernde Flecke zerstreut. Die Gegend des Fussgelenkes und der Fussrücken hingegen sind wieder diffus roth, mit etwa $1 \frac{1}{2} \mathrm{Cm}^{2}$ grossen, feinen, gewöhnlich an einem Rande baftenden Schüppchen bedeckt. Zwischen den Zehen, in den plantären Zehenfurchen löst sich die Hornschichte in grösseren Lamellen los. Die Hornschichte der Ferse ist verdickt und etwas gesprungen.

Die Haut ist nirgends infiltrirt, hat jedoch viel an Elasticitat eingebüsst. Dies Letztere ist aber wohl zum Theile dem vorgeschrittenen Alter des Patienten zuzuschreiben. Sie ist bloss im Gesichte leicht gedunsen und hemmt hier ein wenig das Spiel der mimischen Muskeln. Die Abschuppung ist sehr reichlich und die losgestossenen Schüppchen sammeln sich schon in der kurzen Zeit, welche die Untersuchung im Ambulatorium in Anspruch nimmt, in ziemlicher Menge auf dem Untersuchungstische an. Fährt man mit der Hand in etwas rauher Weise über die Hautfl̈che hinweg, dann steigen kleine Schuppenwölkchen auf.

Die Conjunctiven sind leicht injicirt, die Schleimhaut des Mundes, des Rachens und der Nase normal. Innere Organe gesund. Temperatur normal, Puls 54, Athmung 12 in der Minute. Urin normal.

Algemeinbetinden und Kraftezustand gut. Jucken tritt nur zeitweise und in sehr gexingem Masse auf.

Therapie. Bäder und 5\% Borsalbe. 24. April. Die Röthe und die Abschuppung hat im Gesichte, am Halse und an den oberen Partien des Rückens und der Brust etwas abgenommen. Sehr ausgesprochen sind aber noch immer die weissen Schüppchenmassen im Schnurrbart und an der Haargrenze. Auf der Brust und auf dem Rücken ist neben der fein zersprengten Hornschichte noch Folgendes zu sehen: in den meisten Follikelmündungen hat sich ein hartes, grauweisses, wenig hervorstehendes Hornpfröpfehen gebildet, um welches herum die Haut nicht röther ist, als die der Nachbarschaft. Therapie dieselbe.

29. A pril. Die Gegend um die Nase herum ist leicht angeschwollen, leicht ödematös, lebhaft roth; die Grenzen der Anschwellung verwaschen, die Temperatur derselben etwas gesteigert. Auf dieser Anschwellung befindet sich eine Anzahl erbsengrosser, dunkelgrünlicher, runder, stark adhärirender Borken, welche aus dem Eintrocknen gleich grosser eitriger Bläschen hervorgegangen sind. Beim Abheben der Börkchen bluten die Stellen leicht.

Therapie. Waschungen mit Seife und warmem Wasser, kühle Leinsamenmehlumschläge; später weisse Präcipitatsalbe. 
5. Mai. Die meisten Börkchen sind abgefallen, an ihrer Stelle Restitutio ad integrum. Die Anschwellung in der Nasengegend ist verschwunden. Am Rücken, im Gesichte, am behaarten Kopfe, auf den Extremitäten Röthe und feine Abschuppung. Das Schilfern der Hände und Fùsse ist in geringerem Grade vorhanden; die Hornschichte der Ferse verdickt. Auf der Brust ist die Schilferung und Röthe beinahe ganz verschwunden, bloss in den Follikelmündungen die erwähnten kleinen, sehr wenig prominenten Hornpfröpfchen und beim Kratzen erscheinen silberne Streifen. Seit zwei Tagen Schmerzhaftigkeit des linken Sprunggelenkes; die Gegend desselben ist etwas röther als rechterseits und leicht angeschwollen.

Therapie. Priessnitz-Umschläge. Ruhe.

Im Verlaufe der nächsten zwei Wochen verliert sich diese Anschwellung und die Schmerzhaftigkeit. Der übrige Zustand bleibt beinahe unverändert.

Nach dieser Zeit sah ich den Patienten, der inzwischen wieder seiner Arbeit nachgegangen war, in unregelmässigen und weiter aus. einander liegenden Zeiträumen. Im Laufe des nächsten Monates (Juni) war Haarausfall und Nagelveränderungen aufgetreten.

18. Juli. Seit einigen Wochen besteht ziemlich starker Haarausfall, besonders in der Schläfegegend. Der Haarausfall ist nicht ganz gleichmässig; er befällt einzelne Flecken in stärkerer Weise (en clairières). Dies ist besonders in der Schläfegegend ausgesprochen. Dabei besteht eine starke Pityriasis capitis. Eine ziemlich starke weisse Schuppenauflagerung findet sich noch im äusseren Theile des Schnurrbartes, auf beiden Ohrmuscheln und am Kinn. Das Gesicht sonst von normaler Farbe und glatt. Auf beiden Schultern die Haut leicht infiltrirt, blassroth, von feinen, stark adhärirenden Schüppchen bedeckt.

Auf der Haut der. Brust in der Höhe des Corpus sterni einige gewöhnliche Comedonen und einige nagelgrosse, braunrothe, schuppende, leicht infiltrirte Flecken; ähnliche Flecke in der Regio epi- und hypogastrica. Linige enger aneinander stehende Flecke am Rücken. In der Gegend der Schulterblätter je ein blassrother, fein gerunzelter und leicht schuppender Fleck von unregelmässiger Form. Zwischen den Flecken die Haut von normaler Farbe, aber beim Darüberfahren mit den Nägeln noch leicht schuppend. Die Haut beider Oberarme kaum etwas dunkler als die normale Hautfarbe; sie fühlt sich etwas rauh an, schilfert leicht, mit dem Fingernagel kann man leicht silberne Furchen auf ihr hervorbringen. Bei stärkerem Kratzen steigen noch feine Schuppenwölkchen auf. Dieser Zustand ist auf den Vorderarmen noch mehr ausgesprochen. Die Streckfläche der Ellbogen ist grob gefurcht, etwas rötber als die Nachbarschaft, mit dickerer Hornschichte bedeckt und sehr leicht infiltrirt. Die Haut beider Handrücken und an der Streckseite der ersten Phalangealgelenke ist roth, die Hautfurchen sind hier stärker entwickelt, mit silberartigen, ziemlich adhärirenden Schüppchen bedeckt, stellenweise etwas infiltrirt. Die Röthe ist an diesen Stellen nicht scharf 
begrenzt. A uf einzelnen der zweiten oder dritten Phalangen bemerkt man einen kleinen, röthlichen Fleck, welcher mit Schüppchen bedeckt ist. Alle Fingernägel sind in ihrem hintersten Ábschnitte etwas vor der Lunale in der Richtung einer quer verlaufenden Linie plötzlich eingesunken. Hinter dieser Querlinie ist keine normale Nagelsubstanz zu sehen, sondern eine unebene, querdurchfurchte, schmutzig-graubraune, etwas weichere Nagelsubstanz. Unter den Nägeln hat sich eine dicke, graue Hornmasse angehäuft, welche die Nägel emporhebt. Die Horn. schichte der Handflächen ist stark verdickt, graubraun, an vielen Orten gesprungen und losgelöst. Stellenweise seichte Rhagaden. Am unteren Theile des Bauches und äber den Glütäen einige ähnliche F'lecken, wie auf dem Rücken. Auf den Schenkeln diffuse Röthe und feine Abschuppung. Die Knien ähnlich wie die Lillbogen. Auf den Unterschenkeln zerstreut pfennig- bis thalergrosse, wohl umschriebene, braun-rothe, grob schilfernde Flecke von unregelmässiger Gestalt. Die Hornschicht von der Ferse angefangen bis zu der vorderen Partie der Soble ist. stark verdickt, von Sprüngen durchzogen, graubraun, An den Zehennägeln ähnliche Veränderungen, wie auf den FingernägeIn, aber weniger stark ausgeprägt. Juckgefühl nur sehr gering und tritt bloss zeitweilig auf.

Therapie. Auf den Sohlen Empl. salicyl. saponat,, für den Körper erst Lassar'sche Paste mit 30\% Theer, später Borsalbe.

In weiteren 14 Tagen involviren sich die meisten rothen, schuppenden Flecke, der Haarausfall sistirt. Die Handteller schilfern noch immer und sind roth. Von der vorderen Hälfte der Sohle hat sich die dicke Hornschichte in grösseren Lamellen losgelöst; in der Fersegegend haftet noch immer eine dicke, schmutzig-braune Hornschichte. Auf dem Unterschenkel einige rothe schuppende Flecke.

Gegen Ende August sah ich den Patienten das letzte Mal. Seive Haut war überall von normaler Beschaffenheit, vielleicht etwas trnckener. Bloss die dicke Hornsehicht an der Ferse war noch vorhanden. Es bildet sich normale Nagelsubstanz, welche die abnorme nach vorne schiebt. Das Haar ist wiedergewachsen.

Wir haben es also in diesem Falle mit einem durch Röthung und Schuppung charakterisirten Krankheitsprocess zu thun. Vergleichen wir das Krankheitsbild und den Krankheitsverlauf, wie sie oben dargestellt wurden, mit dem Bilde, welches Kaposi von der Hebra'schen Pityriasis rubra entwirft, so wird es bald klar, dass unser Fall trotz rollkommener Congruenz der objectiven Symptome in Hinblick auf den Verlauf nicht als Pityriasis rubra im Hebra'schen Sinne aufufassen ist.

Die letztere ist dadurch charakterisirt, dass bei derselben "gar keine anderweitige Proruptionsform, weder Knötchen oder 
Bläschen, noch Pusteln, sondern immer einzig und allein vom Beginne an, wie während ihres ganzen Verlaufes, nur Röthung und Schuppung der Haut vorhanden ist." (Kaposi, Hautkrankheiten 1887 , S. 449).

Dabei erscheint die Haut allüberall „lebhaft bis blauroth .... ihre Epidermis in feinen, kleinen Schüppchen, oder in etwas grösseren, dünneren Lamellen sich loslösend.... Die Gesichtshaut von derselben Beschaffenheit und ebenso die des behaarten Kopfes, wäbrend Flachhand und Fusssoble entweder blass oder injicirt, dabei mit einer glänzenden dickeren Epidermisauflagerung versehen sind. Die Hauttemperatur ist erhöht. Auf Druck blasst die Röthe ab, mit Zurücklassung einer gelblichen Tingirung. Subjectiv empfinden die Kranken sehr mässiges Jucken und fortwährend die Empfindung des Fröstelns. Die Krankheit entsteht ohne nachweisbare Veranlassungen und ohne bekannte Vorläufer gleichzeitig an mehreren oder vielen Körperstellen, namentlich den Gelenksbeugen und breitet sich binnen wenigen Monaten, ein bis zwei Jahren über den ganzen Körper aus. Der Verlauf erstreckt sich auf viele Jahre und zeigt niemals eine Aenderung im Sinne der Rückbildung." Im Verlaufe einiger Jahre kommt es zu stellenweiser Verdickung der allgemeinen Decke, theils durch ödematöse Anschwellung, theils durch etwas massigere Schuppenauflagerung. ${ }^{1}$ ) Noch später entwickeln sich atrophische Erscheinungen, die Haut wird dünn, wie zu enge, der Panniculus adiposus schwindet, die Haare fallen aus, die Nägel degeneriren, es entwickeln sich an den stramm angezogenen Hautstellen Einrisse und Geschwüre. Dabei hat die Gesammternährung bedeutende Einbusse erlitten; es stellt sich allgemeiner Marasmus ein, an welchem oder an einer intercurrirenden Krankheit die meisten mit Pityriasis rubra behafteten Kranken nach mehrjährigen Leiden zu Grunde gehen.

Was demnach den mitgetheilten Fall, trotz der beinahe vollkommenen Uebereinstimmung der Hautveränderungen mit

1) In dem ursprünglichen, von Hebra entworfenen Krankheitsbilde geschieht einer Verdickung der allgemeinen Decke keine Erwähnung. 
denen der früheren Stadien der Hebra'schen Pityriasis rubra von der letzteren unterscheidet, ist der im Gegensatze zu dem chronischen, insidiösen, malignen Charakter der Pityriasis rubra rasche und benigne Verlauf.

Die Hebra'sche Pityriasis rubra stellt aber bloss einen Typus der mit Röthung und Schuppung der Haut einhergehenden Erkrankungen dar und zwar einen seltenen. Es wurden von französischen, englischen und amerikanischen Autoren unter verschiedenen Namen Affectionen beschrieben, welche mit Hautveränderungen, die in Röthung und Schuppung bestehen, aber mit einem ron dem obigen und auch unter einander verschiedenen Verlaufe einhergehen. Diese Fälle sind von französischen Dermatologen, in erster Reihe von Brocq, gesammelt und gesichtet worden. Derselbe Autor hat es auch unternommen, die betreffenden Fälle nach bestimmten Typen zu ordnen. Wollen wir also unserem Falle die ihm entsprechende Diagnose geben, so müssen wir auch die von Brocq aufgestellten Typen zum Vergleiche heranziehen.

In Deutschland und Oesterreich-Ungarn hat die Frage von der Constitution der Gruppe der mit Röthung und Schuppung einhergehenden Krankheiten bis in die allerletzte Zeit geruht. Meines Wissens ist der Artikel Jadas obns, auf den ich noch weiter unten zurückkehre, die einzige grössere deutsche Arbeit über diese Frage. Es erscheint daher nicht überflüssig, den Standpunkt der Franzosen in dieser Frage kurz zu präcisiren. In den folgenden Zeilen will ich dies unternehwen, indem ich dabei insbesondere Brocq's und Besnier's Publicationen berücksichtige. ${ }^{\mathbf{1}}$ )

Beide Autoren erkennen die Hebra'sche Pityriasis rubra an. Neben der Pityriasis rubra Typus Hebra beschreiben sie aber noch andere Typen von mit Röthe und Schuppung einhergehenden Krankheiten. Alle diese fasst Besnier unter dem gemeinschaftlichen Namen "erythrodermies exfoliantes" zusammen. Diese Gruppe ist nicht homogen, die in ihr ent-

') Brocq. Archives générales de médecine. 1884. Intern. dermatolog. Congress. Paris 1889 und Traitement des maladies de la peau. 1892. Besnier in der Uebers. des Kaposi'schen Lehrbuches. Band I. p. 617.1891. 
haltenen Processe zum grössten Theile noch nicht genügend gekannt; es kann also nicht davon die Rede sein, die Constitution der Gruppe, so wie sie die französischen Autoren gegeben, als eine definitive anzunehmen. Und auch die genannten Autoren betrachten sie nicht als eine solche. Aber selbst die provisorische Ordnung der hieher gehörigen Formen war sowohl in praktischer Hinsicht, als auch in Bezug auf das weitere, eingehendere wissenschaftliche Studium dieser Processe von hoher Bedeutung.

Brocq stellt gewisse Processe abseits von den eigentlichen exfoliativen Erythrodermien. Diese sind a) die von Besnier als Érythrodermies exfoliantes secondaires unterschiedenen Processe, welche sich dem Verlaufe verschiedener Dermatosen, wie Psoriasis, Ekzem, Lichen, Pemphigus, anschliessen können. Diese werden entweder durch locale Application von gewissen Stoffen und Medicamenten, oder durch die Injection gewisser Substanzen hervorgerufen. Ein grosser Theil der Fälle des später zu erwähnenden Erythema scarlatiniforme hat diesen Ursprung.

Eine Anzahl anderer Fälle, welche Besnier Érythrodermies exfoliantes secondaires terminales nent (Bazin's Herpétides exfoliatrices), entwickeln sich nicht bei jedem beliebigen Falle einer grossen Dermatose, wie die frïheren, sondern bloss bei herabgekommenen Individuen, nachdem die ursprüngliche Dermatose (eine der oben genannten) schon lange Zeit bestanden. Sie haben eine jahrelange Dauer, compliciren sich mit visceralen Veränderungen und enden mit dem Tode.

b) Die Lymphodermia perniciosa (Kaposi) und gewisse generalisirte, ekzematiforme Varietäten der Mycosis fungoides. (Die dermatites lymphoides von Besnier.) Bei diesen ist die Haut infltrirt, verdickt; der starke Pruritus ruft ekzematiforme Veränderungen hervor und die Exfoliation ist gering. Es gibt jedoch gewisse Formen von Erythrodermien, welche dem prämykotischen Stadium (Besnier) angehören, Jahre hindurch bestehen können und bei welchen die Differentiation von den eigentlichen Erythrodermien eine viel schwierigere ist. 
c) Die Pityriasis rubra pilaris Devergie, jene bekannte, von der Wiener Schule zu dem Lichen gezählte Krankheit.

d) Endlich müssen wir hieher stellen die von Besnier angeführten Dermatitiden, welche sich tiefen Fiterungsprocessen anschliessen. Sie sind ekzematiform, sehr pruriginös, gehen mit Oedem und Infiltration der Haut und geringer Abschuppung einher.

Die unter b), c) und d) angeführten Krankheitsprocesse figuriren bei Besnier in der Gruppe der chronischen, idiopathischen, primitiven Erythrodermien, ohne dass aber dabei an einen engeren Zusammenhang derselben mit den übrigen in diese Gruppe gestellten Processen, den eigentlichen exfoliativen Erythrodermien mit chronischem Verlaufe, gedacht würde. ${ }^{1}$ )

In derselben Weise wird die folgende Classe von Besnier bei den a,cuten und subacuten, idiopathischen, primitiven, exfoliativen Erythrodermien angefüht:

e) Initiale, exfoliative Dermatitiden, unter deren Erscheinungsweise manchmal das Ekzem, die Psoriasis, der Lichen und Pemphigus etc. paratypisch auftreten.

Ausser den sub a), b), c), d), e) angeführten Processen gibt es noch gewisse mit Röthung und Schuppung einhergehenàe Krankheiten, welche als eigentliche exfoliative Dermatitiden, oder wie Brocq sie nennt, als Eruptions généralisées rouges et desquamatives primitives unterschieden werden müssen. Dieses sind die folgenden:

A. Das Erythema scarlatiniforme desquamativum oder Dermatitis exfoliativa acuta benigna. Das Erythema scarlatiniforme tritt plötzlich und mit Fieber verschiedener Intensität auf. Kranke, die mehreremale davon heimgesucht wurden, berichten auch über gewisse prodromale Erscheinungen. Das Fieber verschwindet rasch und die Kranken bleiben während des grössten Theiles ihrer Erkrankung fieber-

1) Besnier theilt nämlich sämmtliche primitive Erythrodermien nach ihrem mehr acuten oder chronischen Verlauf in zwei grosse Gruppen, wobei natüricherweise Processe verschiedener Bedeutung neben einander gerathen. Diese Eintheilung ist bloss eine provisorische, artificielle und bezweckt eine Erleichterung des weiteren Studiums. 
frei. Die Eruption tritt gewöhnlich an mehreren Stellen der Körperoberfläche auf und verbreitet sich allmälig über dieselbe, wobei sie endlich dieselbe in ihrer ganzen Ausdehnung einnehmen oder grössere Partien frei lassen kann. Es treten verschieden geformte Flecken, zumeist von Scharlachröthe auf, deren Farbe unter dem Fingerdrucke schwindet. Häufig bemerkt man, dass die Injection der Gefässe mit Vorliebe um die Follikelmündungen herum auftritt. Im Anfange gesellen sich zuweilen mehr oder weniger ausgebreitete Oedeme hinzu. Tiefere Veränderunger des Hautgewebes (ekzematoide Veränderungen, Phlegmasien, Necrosen, Folliculitiden und Hydroadenitiden) compliciren das Erythema scarlatiniforme bloss in seinen schwereren, mit länger dauerndem Fieber einhergehenden Formen und in seinen vorgeschrittenen Stadien. Die subjectiven Symptome (Brennen, Jucken) spielen im ganzen Verlaufe bloss eine untergeordnete Rolle; sie sind bloss im febrilen Anfangsstadium stärker ausgeprägt. Die Schleimhaut des Mundes und Rachens kann besonders im Anfange stark geröthet sein und desquamiren; viel weniger intensir pflegen die Nasenschleimhaut und die Conjunctiven befallen zu sein.

Die Abschuppung steht bei dem Erythema scarlatiniforme im Vordergrunde des Interesses in Folge ihres frühen Auftretens, ihrer Dauer und Constanz und Intensität. Das frühe Auftreten (3-4 Tage nach dem ersten Erscheinen der Röthe) der Schuppung, ihr gleichzeitiges Bestehen mit dem Erythema ist neben dem Mangel der Contagiosität, dem langen Persistiren des Erythems etc. eines der Hauptmerkmale, welche das Erythema scarlatiniforme von den Exanthemen, bei welchen die Desquamation der Röthe nachfolgt, unterscheidet. Die Desquamation ist sehr reichlich, sich immerfort erneuernd. Sie besteht aus feinen, weissen Schuppen von verschiedener Grösse, kleienförmig im Gesicht, lamellös auf den Extremitäten, handschuh- resp. sandalenförmig auf den Händen resp. Füssen. An den Nägeln kann je nach der Intensität des Falles mehr oder weniger ausgeprägte Furchung auftreten, ja es kann sogar zum Ausfallen der Nägel, ebenso auch zu dem der Haare knmmen. Die Dauer des Erythema scarlatiniforme ist gewöhnlich. zwei bis sechs Wochen, zwei Monate Maximum. Fälle mit 
längerer Daner treten aus dem ursprünglichen Rahmen des Erythema scarlatiniforme heraus und nähern sich den eigentlichen Dermien oder Dermatitiden.

Eine weitere charakteristische Eigenschaft dieser Krankheit ist ihre ausserordentliche Recidivfähigkeit. Doch können auch Fälle ohne Recidive zur Beobachtung kommen.

Besnier unterscheidet nach der Dauer der Fälle eine benigne, acute Form, und eine subacute, prolongirte Form des E. scarlatiniforme. Die letztere geht ohne scharfe Grenze in die leichtere Form der grossen exfoliativen Dermititiden über und hat häufig einen schwereren Verlauf.

B. Eigentliche oder subacute Dermatitis exfoliativa generalisata (Brocq). Diese ist eine allgemeine Erkrankung, welche nicht contagiös zu sein scheint und hat einen cyklischen Verlauf. (Periode der Entwicklung, des Bestandes und der Rüickbildung.) In ihren ersten beiden Perioden ist sie febril und das Fieber zeigt einen continuirlichen, remittirenden Charakter. Der Anfang ist manchmal plötzlich, zumeist insidiös. Es treten ein oder mehrere rothe, pruriginöse Flecken auf, welche sich in zwei bis zehn Tagen über den ganzen Körper verbreiten. Hat sich die Krankheit etablirt, dann ist die ganze Haut intensiv roth, mehr oder weniger infiltrirt, häufig wie gespannt. Einige Tage nach Erscheinen der Röthe tritt die Exfoliation in Form feiner, perlmutterartiger, trockener, gewöhnlich 2-3 Centimeter breiter, $1-1 \frac{1}{2}$ Centimeter langer Schuppen auf, welche sich zuweilen dachziegelförmig decken.

Die Haare fallen immer vollkommen oder zum Theile aus; sebr oft auch die Nägel, welche zumindest tiefe Furchen aufweisen. In einzelnen Perioden der Krankheit und an einzelnen Stellen des Körpers, besonders an den Gelenksbeugen, kann sich Nässen entwickeln. Dieses verbreitet einen üblen Geruch. Desgleichen können sich manchmal pemphigoide Blasen, Pusteln, Furunkeln, Seborrhoe circumpiläre Hornkegel entwickeln. Jucken besteht beinahe constant, oft auch Brennen. Die Kranken frösteln, so wie man sie entblösst. Die Schleimhäute können mitbefallen sein. Die Lymphdrüsen sind häufig vergrössert. 
Die benignen Formen laufen in drei bis vier Monaten ab, die schwereren in fünf bis sechs Monaten; treten schwere Complicationen auf (Anthrax, Abscesse, Phlegmonen, Iritis, Taubheit, arthritische Erscheinungen, Complicationen von Seite des Herzens, Paraplegien etc.), oder kommen mehrere Schübe nacheinander, dann benöthigt die Krankheit 6-10 Monate oder auch ein Jahr bis zur rollkommenen Genesung. Sie kann auch gegen den dritten oder vierten Monat mit Diarrhoe, Marasmus oder Complicationen hauptsächlich von Seite der Lunge letal endigen.

Recidive und Rückfälle sind möglich. Oft bleibt eine Pigmentation der allgemeinen Decke lange nach Ablauf der Krankheit bestehen.

C. In der Gruppe der exfoliativen Dermatitiden führt Brocq auch die von Ritter von Ritterheim zuerst beschriebene Dermatitis exfoliativa der Säuglinge an. Dies ist eine acute, nicht contagiöse Affection, welche durch eine Anfangs localisirte, später generalisirte Röthe und Desquamation charakterisirt wird, im Verlaufe der ersten bis dritten Woche nach der Geburt auftritt und in $50 \%$ der Fälle sehr rasch zum Tode führt.

Ausser den bisher erwäbnten acuten und subacuten exfoliativen, primitiven, idiopathischen Dermatitiden gibt es noch solche, welche sich durch chronischen Verlauf auszeichnen. Diese fasst Besnier unter dem Titel Erythrodermies à debut variable, évoluant secondairement à la manière du pityriasis rubra ou des dermatites exfoliatrices zusammen.

Diese Gruppe von Krankheitsprocessen wird, wie B e s n i e r darlegt, charakterisirt durch die ausserordentliche Abwechslung der Hautveränderungen in ihrem Anfangsstadium und die ausserordentliche Ungleichheit der Daxer ihrer ersten Entwicklungsperioden. Die Hautveränderungen in ihrem Anfangsstadium haben nichts Typisches. Bald erscheinen, nachdem häufig ein insidiöses, bloss durch Jucken charakterisirtes Stadium vorangegangen war, Läsionen, welche Resultate des Kratzens zu sein und dem banalen Ekzemtypus, dem seborrhoischen oder akneiformen Ekzema anzugehören scheinen; 
bald wieder sind es einfache erythematöse Flecke, Nodositäten obne bestimmte Bedeutung, eine Dermatitis, welche Anfangs um eine frische oder ältere Continuitätstrennung herum localisirt ist etc. etc. Nachdem dieses larvirte Stadium mehrere Wochen oder Monate gedauert hat, nimmt die Affection eine bestimmtere Form an, indem sie, je nachdem die Erscheinungen der Dermatitis mehri oder weniger Acuität besitzen und je nachdem die Desquamation gross- oder feinlamellös ist, dem Typus der exfoliativen Dermatitis oder dem der Pityriasis rubra (Hebra) in ihren vorgeschritteneren Stadien folgen.

In dieser Gruppe von in ihrem Wesen ganz dunklen, ihrem Symptomencomplex und ihrer Verlaufsweise nach noch viel zu wenig studirten Processen hat Brocq versucht, gewisse Typen aufzustellen. Er sowohl als Besnier glauben jedoch, dass bloss neuere klinische und histologische Beobachtungen eine definitive Ordnung dieser exfoliativen Dermatitiden ermöglichen werden. Wir fahren nun in der Beschreibung der eigentlichen exfoliativen Dermatitiden fort, indem wir bei den chronischen Formen derselben die von Brocq aufgestellten Typen aufzählen werden.

D. Dermatitis exfoliativa generalisata chronica. Dieselbe ist charakterisirt 1. durch ein sehr langes Anfangsstadium, während welcher die Affection ein Ekzema simulirt und sich allmälig ausbreitet; 2. eine ebenfalls sehr lange Periode des Bestandes. Während derselben bietet die Affection die hauptsächlichen Charaktere der typischen Dermatitis exfoliativa; generalisirte Röthe und Infiltration der Lederhaut, ununterbrochene und reichliche Desquamation in grossen Lamellen, Haarausfall, Veränderungen und Ausfall der Nägel, Anschwellung der Lymphdrüsen, Complication, wie z. B. Abscesse, Amblyopie, Taubheit etc. 3. Sie wird endiich charakterisirt durch ein Rückbildungsstadium, in dessen Verlaufe die Röthe und Desquamation allmälig verschwinden, indem sie eine bräunliche Pigmentirung zurücklassen, welche nur sehr langsam rerschwindet. Dauer: mehrere Jahre.

E. Die Pityriasis rubra Typus Hebra, Pityriasis rubra chronica gravis (Brocq), deren Beschreibung wir oben schon gegeben haben. 
Neben dieser Form der Pityriasis rubra wirft Brocq noch die Frage von der Existenz zweier weiterer Formen derselben auf, es ganz den weiteren Beobachtungen anheimstellend, darüber zu entscheiden, ob diese als selbständige Krankheitsentitäten anzuerkennen sind oder nicht. Diese sind:

F. Die Pityriasis rubra subacuta benigna(Vidal), welche charakterisirt ist durch eine generalisirte, pruriginöse Röthe, eine feine, kleienförmige Abschuppung, eine leichte Steigerung der Temperatur während des Anfangsstadiums und der Periode des Bestandes, durch die Integrität der Nägel und Haare ${ }^{1}$ ) nnd durch das günstige Ende in Heilung im Verlaufe von 6-7 Monaten.

G. Die Pityriasis rubra chronica benigna. Dieselbe hat gemeinsame Züge mit den Lichenarten, mit der Psoriasis und der Pityriasis rubra. Bei dieser tritt eine grosse Menge "quasi-papulöser", jedoch nicht besonders erhabener, squamöser, rother Elemente von der Grösse eines kieinen Stecknadelkopfes bis zu der einer Linse und darüber auf, welche anfangs isolirt stehen und endlich zu rothen Flecken confluiren. Man sieht sie besonders am Halse, an den Seitenflächen des Stammes, an den Vorderarmen, an den Aussenseiten der Unterextremitäten. An manchen Stellen, z. B. auf der Brust, besteht diffuse Röthe mit einer Art von Rissigkeit („craquelures“), welche an ein abortives Ekzem erinnert.

Die Schuppen sind fein, kleienförmig, weiss, adbärent, psoriasiform, besonders über den Papeln. Man kann jedoch an diesen Stellen durch Kratzen nicht die rothe, glatte, glänzende Oberfläche mit der hämorrhagischen Punktirung der Psoriasis darstellen.

Die Eruption ist pruriginös; sie braucht mehrere Monate bis ein Jahr, um sich zu generalisiren, und auch dann sind manche Stellen des Körpers - wie das Gesicht - noch wenig befallen.

Nach mehreren Jahren wird die Röthe dunkler und zu gleicher Zeit wird die Haut dünner und faltig. Aber auch in

†) Die Veränderungen der Nägel und Haare treten bei der Hebra'schen Pityriasis rubra erst in späteren Stadien der Entwicklung auf. 
dieser vorgeschrittenen Periode der Krankheit ist der Allgemeinzustand ein befriedigender. Man findet immer noch einzelne "quasi-papulöse" Elemente und einzelne ganz kleine normale Hautstellen.

Die nach Brocq mitgetheilten Typen umfassen jedoch noch immer nicht alle Formen der exfoliativen Dermatitiden; es gibt noch Fälle - Besnier führt sie an - welche nach larvirtem Anfange, wie er oben beschrieben wurde, später ganz die Alluren der Pityriasis rubra Typus Hebra annehmen. Nichts charakterisirt die objective und skeptische Weise, wie die Franzosen diese Frage behandeln, besser, als der Standpunkt, den Besnier denjenigen Processen gegenüber einnimmt, welche er unter dem Titel Erythrodermies exfoliantes secondaires terminales (s. o.) zusammenfasst. Er wirft hier die Frage auf, ob man den Ansgang gewisser protrahirter Psoriasis-, Fkzem-, Lichen- etc. Fälle in universelle exfoliative Erythrodermie als fatale Entwicklungsphase dieser Krankheiten oder vielmehr als neue, als complicirende Erkrankung betrachten soll.

Aus dem bisher Vorgetragenen geht mit Deutlichkeit hervor, dass die französischen Autoren unter den primitiven mit Röthung und Schuppung einhergehenden Krankbeitsprocessen das mit rascherem Verlaufe verbundene Erythema scarlatiniforme und die Dermatitis exfoliativa generalisata subacuta als wohlcharakterisirte Krankbeiten nnterscheiden. Hinsichtlich der exfoliativen Dermatitiden, welche chronisch verlaufen, ist ihr Standpunkt der, dass es zur Zeit unmöglich ist, eine definitive Ordnung zu schaffen unter den hieher gehörigen Processen, für welche - wie Besnier erklärt - es nöthig wäre, beinahe ebensoviel nosographische Fächer zu errichten, als es Einzelfälle gibt. Diese Processe sind dem weiteren Studium zu unterziehen. Die mit verschiedener Intensitä der entzündlichen Erscheinungen, der Röthe, der Infiltration der Schuppung einhergehenden, sich durch ihre Anfangserscheinungen, ihren Verlauf, ibre Allgemeinsymptome etc. von einander unterscheidenden Processe haben sie versucht, in einige $\mathrm{pr}$ ovis or is ch e Gruppen einzureihen, deren charakteristische Merkmale wir oben mitgetheilt haben. 
In einer der Pityriasis rubra gewidmeten Studie folgt Jadassohn ${ }^{3}$ ) insoferne den Franzosen, als er die Dermatitis exfoliativa subacuta generalisata und das Erythema scarlatiniforme, und zwar das letztere mit Vorbehalt, anerkennt. Was die chronisch verlaufenden, mit Erythem und Schuppong verbundenen Processe betrifft, ist sein Standpunkt der, dass er auf Grund einer Anzahl von aus der Literatur gesammelten Krankengeschichten annimmt, hinsichtlich der Existenz und Selbständigkeit des von Hebra geschilderten und als Pityriasis rubra bezeichneten Krankheitsprocesses sei ein Zweifel nicht berechtigt. Es sei jedoch auf Grund neuerer Erfahrungen das Bild derselben mehr oder weniger wesentlich zu erweitern und zu verändern. Dies geschieht nun in der Weise, dass in dem ursprünglichen Symptomencomplex neben dem Mangel oder der ganz unbedeutenden Infiltration, neben der kleienförmigen Schuppung, neben der Trockenheit des Processes, neben der geringen Intensität des Juckens, neben dem Ausgange in Atrophie erhebliche Hautinfiltration, grosslamellöse, schildförmige Desquamation, Nässen, intensives Jucken und Restitutio ad integrum Platz ifnden, neben dem gleichmässig chronischen, nicht remittirenden Verlauf, neben dem Kräfteverfall, neben dem Marasmus kürzerer Verlauf, mit Remissionen und Recidiven, guter Allgemeinzustand und vollständige Heilung figuriren. Hiedurch sind aber die Grenzen der "Pityriasis rubra" so weit gesteckt worden, dass sämmtliche chronische desquamative Erythrodermien darin Platz finden, und $\mathrm{J}$ ad a ssohn kann daher die Nothwendigkeit zu einer „noch weiter gehenden Scheidung in Pityriasis rubra subacuta und Dermatitis exfoliativa chronica" leugnen.

Nun kann ja die Möglichkeit, dass die hier in Betracht kommenden chronischen Erythrodermien insgesammt einmal als in eine gemeinsame Gruppe gehörig erkannt werden würden, heute nicht geleugnet werden. Wir sind noch zu wenig in das Wesen dieser Processe eingedrungen, um solche Fragen definitiv im bejahenden oder verneinenden Sinne beantworten zu können.

1) Archiv für Dermatologie. 1891 und 1892. 
Wird aber eines Tages erkannt werden, dass diese Pro. cesse auf Grund einer gemeinsamen Pathogenie oder Aetiologie mit einander in enge Verbindung gebracht werden müssen, dann wird man doch kaum auf die so abwechslungsreichen Bilder die Etikette der ursprünglichen Hebra'schen Pityriasis rubra heften können. Die letztere bezöge sich ja auch dann nur auf eine bestimmte und zwar geringe Anzahl von Fällen. Wir hätten es eben nicht so sehr mit einer Erweiterung des alten Hebra'schen Begriffes zu thun, sondern vielmehr mit einem ganz neuen.

Wie gesagt ist aber eine Discussion in dieser Richtung heute noch verfrüht. Keineswegs kann heute die andere Möglichkeit geleugnet werden, dass sich hinter den so verschiedenen Bildern auch verschiedene Krankheiten verbergen. Wollen wir in dieser Gruppe Ordnung schaffen, dann ist es bei dem heutigen Stande unseres Wissens, bei der klinischen Untersuchungsmethode, auf welche wir uns bei diesen Fragen in erster Reihe stützen, geboten, bis auf weiteres an den schon geschaffenen Typen strenge festzubalten, uns bloss unwesentliche Modificationen an ihnen zu erlauben und die in ihrer klinischen Erscheinungsweise und in ihrem Verlaufe verschiedenen Fälle in verschiedenen, wenn auch nur provisorischen Gruppen unterzubringen. Eben darum ist das Bestreben der Franzosen, „zu rubriciren und zu classificiren“, ein berechtigtes. Diese Classificirung, welche - wie ja aus Besnier's Darstellung zur Genüge hervorgeht - heute eine noch sehr unvollkommene ist, ist unter den obwaltenden Verhältnissen der Untersuchungsmethoden geradezu ein Gebot der Nothwendigkeit. Bloss dadurch können wir uns vor dem Irrthume hüten, Processe von verschiedener Bedeutung in eine zu enge Verbindung zu bringen. Fine definitive Ordnung dieser Processe können wir aber erst vornehmen, wemn wir besser in das Wesen derselben eingedrungen sein werden.

Wie ich angedeutet habe, hat es Jadassohn unternommen, schon auf Grund unserer bisherigen Erfahrungen dem alten Hebra'schen Pityriasis rubra-Begriff unter dem Titel einer mehr oder minder wesentlichen Erweiterung und Veränderung desselben, einen neuen zu substituiren. Ich war bestrebt, in 
der obigen kurzen Argumentation dieses Unternehmen als ein solches darzustellen, welches durch unsere heutigen Kenntnisse noch nicht gestützt werden kann und zumindest als verfrüht anzusehen ist.

Nach diesem kurzen Ueberblick über den heutigen Stand der Frage von den exfoliativen Erythrodermien kehren wir zu unserem Falle zurück. Es bedarf, wie ich glaube, keiner eingehenden Besprechung, um auszuschliessen, dass wir es hier mit irgend einer Form der chronischen exfoliativen Erythrodermien zu thun hätten. Die letzteren bieten ausser ihrem langwierigen Verlauf schon durch ihre schweren Allgemeinerscheinungen genügende Anhaltspunkte zur Differenciation gegenüber unserem Falle. Es wäre auch ein müssiges Unterfangen, die Unterscheidungsmerkmale hier noch weiter und detaillirter zu verfolgen. Ein Blick auf die Krankengeschichte und auf die oben mitgetheilten kurzen Charakteristiken der in Betracht kommenden. Processe lässt die Differenzen klar hervortreten. Einer eingehenderen Discussion müssen wir aber die Dermatitis generalisata subacuta, das Erythema scarlatiniforme und die Pityriasis rubra benigna subacuta unterziehen. Gegen die Annahme einer Dermatitis exfoliativa subacuta spricht in unserem Falle der Mangel eines cyklischen Verlaufes, der afebrile Zustand des Kranken, während der ganzen Beobachtung, die Ausbreitung des Processes, welche rascher vor sich ging, als dies bei der D. e. g. gewöhnlich der Fall ist; des weiteren der Mangel einer Infiltration und Anschwellung des Teguments. Und doch gibt es einzelne Symptome, welche den Fall der Derm. exf. gen. etwas näher bringen. So z. B. die Dauer, der Haarausfall, die Veränderung der Nägel: Thatsachen, welche bei dem Erythema scarlatiniforme, mit welchem unser Fall die meiste Aehnlichkeit besitzt und zu welchern wir ihn auch stellen, seltener beobachtet werden. Es ist aber hervorzuheben, dass zwischen dem Erythema scarlatiniforme und der Dermatitis exfol. generalisata subacuta allmälige Uebergänge beobachtet werden und es sind gerade solche Fälle von [Erythema scarlatiniforme, wie der unsrige, d. $h$. Fälle mit protrahirtem Verlaufe, Ausfall der Haare und Nagelveränderungen, durch welche sich das E. scarl. den leichteren 
Fällen der Derm. exf. subacuta nähert. Das Erythema scarlatiniforme wurde auch aus diesem Grunde als eine Derm. exfol. gen. a cuta aufgefasst.

Was spricht nun in unserem Falle für die Diagnose Erythema scarlatiniforme? Vor Allem das plötzliche Auftreten, die rasche Entwicklung und Ausbreitung des Processes, der Mangel einer nennenswerthen Störung des Allgemeinbefindens, die Abwesenheit des Fiebers. Freilich pflegt das Letztere nur ausnahmsweise, während des Ausbruches der Krankheit zu fehlen; doch ist das Fehlen des Fiebers im Beginne in leichten Fällen schon beobachtet worden, besonders wenn die Hautveränderung nicht generalisirt war. Vielleicht ist es erlaubt, auch unseren Fall, trotz der Universalität des Ausschlages, zu den leichteren zu stellen, da mit Ausnahme des letzteren Umstandes Alles in der Krankengeschichte für eine ganz unbedeutende Erkrankung spricht.

Im übrigen Verlaufe ist jedoch das Erythema scarlatiniforme immer apyretisch; und dieser Eigenschaft entspricht unser Fall vollkommen.

Eine weitcre Thatsache, welche für die Diagnose E. scarl. anzuführen ist, ist das frühe Auftreten, die Dauer und die Intensität der Desquamation. Auch die Form der Schuppen im Gesichte, auf den Extremitäten, Händen und Füssen entspricht vollkommen dem E. scarl. Weniger die mehr kleienförmige, kleinlamellöse des Stammes, wo sie sonst unregelmässiger und in etwas grösseren Lamellen aufzutreten pflegt. Ebenso entspricht der Diagnose E. scarl. die Unbedeutendheit der subjectiven Symptome. Ueber die Dauer des Processes, welche die von Brocq angegebene Maximalzeit um das Doppelte übersteigt, sowie über die Veränderung der Nägel und Haare habe ich mich schon weiter oben ausgesprochen.

Der kürzere Verlauf, der Mangel einer Temperaturerhöhung, die Veränderung der Nägel und der Haarausfall, sowie die geringe Intensität der subjectiven Symptome unterscheiden den obigen Fall von der Pityriasis rubra benigna subacuta (Brocq). Wir müssen denselben demnach ansprechen als einen Fall von Erythema scarlatiniforme mit protrabirtem Verlaufe. 